\title{
Radiopharmaceuticals in the evaluation and treatment of liver lesions
}

\author{
Stefano Fanti $\cdot$ Adil AL-Nahhas
}

Received: 21 February 2014/ Accepted: 15 March 2014/Published online: 3 April 2014

(C) Italian Association of Nuclear Medicine and Molecular Imaging 2014

Radiopharmaceuticals have long been used to study the liver, giving interesting results. Nonetheless, for most indications, radionuclide imaging has not tended to be a first-choice approach, due to the better diagnostic performance of other imaging methods, namely, contrastenhanced computed tomography (ceCT) and ultrasound (US). However, the emergence, in recent years of radiolabelled tracers for use with positron emission tomography (PET) has led to renewed interest in radionuclide imaging of the liver. Applications (both new and more established ones) include the evaluation of hepatocellular carcinoma using choline PET, the use of FDG PET/CT for identifying secondary liver lesions and for localising unknown primary tumours in patients with liver metastases, as well as innovative approaches, such as the use of hybrid PET/ magnetic resonance imaging (PET/MRI) and PET-guided liver biopsies. Furthermore, radioembolisation as a treatment for liver lesions has recently been successfully introduced.

This issue of Clinical and Translational Imaging is devoted to these topics. It has been compiled by a group of experts in this field, some of whom are pioneers and leaders at European and international level. In addition to setting out the rationale and background behind these approaches, they have endeavoured to provide an overview of the current state of the art, comparing their experience with

S. Fanti $(\square)$

Department of Nuclear Medicine, S. Orsola University Hospital,

PAD 30, Via Massarenti 9, 40135 Bologna, Italy

e-mail: stefano.fanti@aosp.bo.it

A. AL-Nahhas

Department of Nuclear Medicine, Imperial College NHS Trust, London, UK that of their contemporaries in an effort to reach to a consensus.

An increasingly important area is that of PET/CT imaging of hepatocellular carcinoma (HCC) using different radiopharmaceuticals. The group of Jean-Noël Talbot in Paris pioneered the use of ${ }^{18} \mathrm{~F}$-fluorocholine $(\mathrm{FCH})$, the fluorinated analogue of choline, for imaging HCC with PET [1], obtaining promising results. It is, in fact, well known that ${ }^{18} \mathrm{~F}$-fluorodeoxyglucose (FDG), the reference PET tracer in oncology, has limitations in the detection of intra-hepatic well-differentiated HCC, and therefore, does not allow accurate study of affected patients. At present, dual-tracer PET with FCH and FDG could constitute a better approach, as the aggressiveness of lesions can vary and some lesions may take up only one tracer. Dual-tracer PET can also be used for disease staging and for predicting recurrence [2] and can therefore be useful in the selection of patients for liver transplantation or HCC tumour resection. Metastases from non-HCC liver malignancies, on the other hand, are not usually FCH avid, while cholangiocarcinoma (CC) can be visualised as a hot focus.

As regards benign liver tumours, it is important from a prognostic point of view to differentiate hepatocellular adenoma, which can degenerate in up to $4 \%$ of lesions from $\mathrm{FNH}$, which is composed of normal hepatocytes occurring in a normal liver: promising results have recently been published which suggest that FCH PET/CT could play a role in differentiating between these two benign lesion types.

Despite the aforementioned limitations of FDG as a radiopharmaceutical for $\mathrm{HCC}$ imaging, there are some indications for the use of this tracer in liver lesion imaging, and this issue is covered by Ilaria Grassi et al. from the Bologna group. The identification of secondary lesions using FDG is undoubtedly the most frequent such 
indication, as metastatic disease accounts for the majority of malignant lesions in the liver and up to $25 \%$ of patients with known solid malignant tumours have hepatic metastases at the time of diagnosis. Liver metastases can originate from numerous primary cancers (colorectal, mammary, lung, pancreatic, gastric, oesophageal, melanoma and sarcoma among others), and the use of FDG $\mathrm{PET} / \mathrm{CT}$ in liver metastases has proved effective for staging purposes, as well as for planning a more correct therapeutic approach to the disease [3].

FDG has also been shown to be useful in $\mathrm{CC}$, in which there is evidence to suggest that it could have roles in staging, metastatic disease assessment and diagnostic management of patients with para-hilar or central lesions not detected through conventional imaging [4].

PET/MRI is definitely the most exciting of the new hybrid approaches to nuclear medicine imaging: Afshin Nasoodi et al., from UCL in London, discuss the use of PET/MRI in identifying and characterising liver lesions. MRI offers high-resolution structural data, superb soft-tissue contrast and a range of acquisition techniques capable of generating functional data. PET/MRI can be used to improve upon the soft-tissue contrast offered by MRI and the biochemical and metabolic information provided by PET, with an overall improvement in test sensitivity and hence increased diagnostic accuracy and improved tissue characterisation. In liver imaging, PET/MRI is superior to stand-alone PET/CT or MRI [5]. PET/MRI can be performed without contrast media and is associated with reduced ionising radiation; the diversity of PET tracers adds further specificity to the technique. In order for PET/ MRI to be viable in clinical routine, however, the imaging workflow and the use of various sequences must be based on patients' specific clinical needs, so as to maximise useful information whilst minimising acquisition time. The next decade will witness the development of more technologically advanced combined PET/MRI machines with further innovations to suit the era of personalised patient management. In the meantime, however, as the authors conclude, PET/MRI remains a prominent research tool for answering complex clinical questions in a selective manner.

The differentiation of benign from malignant liver lesions is another area, in which PET/CT may play an effective role. Swati Rachh et al., from the Tata Memorial Centre in Mumbai, in their comprehensive literature overview, describe the existing results in this field. Benign liver lesions have a high prevalence; therefore, accurate lesion characterisation (in addition to detection) is a very important requirement. However, it is one that anatomical techniques such as US, CT, and MRI struggle to meet. Functional imaging is an additive modality in this setting, aiding in the differentiation of atypical benign vascular and other liver lesions from malignancy, and obviating the need for biopsies and histopathological assessments [6]. MRI is, in general, a more accurate and useful modality than PET for small cystic lesions; small lesions (especially those below $5-10 \mathrm{~mm}$ in size) can be beyond the resolution capacity of PET, while cystic metastases and disease with mucinous histology are also often false negative or show low intensity on FDG imaging, due to these lesions' low tumour cell density. Haemangioma is the most common benign liver tumour and in a small proportion of cases morphological imaging results are indeterminate: FDG PET can differentiate benign atypical haemangioma from metastatic disease, thus avoiding the need for histopathological correlation and therefore, the risk of haemorrhage resulting from biopsy of a benign vascular liver lesion. PET is also useful in indeterminate cases of focal nodular hyperplasia and hepatic adenoma [7].

A completely new approach for studying the liver is PET/CT-guided biopsy. It is a method only recently introduced, and Juliano Cerci et al. from Curitiba, describe the technique and report their experience in this innovative and promising setting. In general, PET/CT-guided biopsy is regarded as a technique that is still evolving, and its value is challenged, given the other imaging-guided biopsy procedures already extensively used in clinical practice. CT is commonly used to guide abdominal biopsies, but some tumours are not visible without intravenous contrast material; MRI can also be used, although open or widebore magnets are typically needed to reach abdominal masses, and biopsy equipment must be MR compatible. Moreover, the information provided by these methods is usually anatomical. PET/CT, on the other hand, provides both anatomical and metabolic information which naturally makes it an attractive prospect not only for application in diagnostic imaging, but also as a means of refining percutaneous interventions. Most notably, it is useful in accessing lesions visible with PET but not with CT, and masses with heterogeneous patterns of FDG uptake (where PET could guide the needle to the part of a lesion with higher metabolic uptake, i.e. the part more likely to contain representative oncological cells) [8].

Finally, radionuclide therapies are becoming increasingly widespread in clinical oncology: Henry Tam et al. from Imperial College, London, review the use of radioembolisation with ${ }^{90}$ Yttrium-microspheres for treating liver lesions. The rationale for radioembolisation rests on evidence that both primary and metastatic liver lesions derive $95 \%$ of their blood supply from the hepatic artery, whereas normal liver parenchyma receives $25 \%$ of its blood supply from the hepatic artery. Therefore, loco-regional treatment of selected liver lobes or segments is feasible. This treatment can be performed using chemotherapeutic agents (transarterial chemoembolisation) or radiopharmaceuticals 
(selective internal radiation therapy). ${ }^{90} \mathrm{Y}$-labelled microscopic resin or glass beads are delivered through intraarterial infusion of selected branches of the hepatic artery; once injected, the radiolabelled microspheres lodge within the tumour vascular bed permanently; accumulating at the edge of tumours, they deliver activity upward of $100 \mathrm{~Gy}$ to tumour tissues [9]. The article provides a complete description of available methodologies, a definitive literature review and an illustration of the authors' experience in this emerging field.

In conclusion, we hope that this issue, in addition to providing an update of the present state of the art in this field and an overview of the available techniques, also offers a glimpse of what the future holds for this exciting area of nuclear medicine.

Conflict of interest Stefano Fanti and Adil AL-Nahhas declare no conflict of interest.

\section{References}

1. Talbot JN, Gutman F, Fartoux L, Grange JD, Ganne N, Kerrou K, Grahek D, Montravers F, Poupon R, Rosmorduc O (2006) PET/CT in patients with hepatocellular carcinoma using $\left[{ }^{18} \mathrm{~F}\right]$ fluorocholine: preliminary comparison with $\left[{ }^{18} \mathrm{~F}\right] \mathrm{FDG}$ PET/CT. Eur J Nucl Med Mol Imaging 33:1285-1289

2. Fartoux L, Balogova S, Nataf V, Kerrou K, Huchet V, Rosmorduc O, Talbot JN (2012) A pilot comparison of ${ }^{18} \mathrm{~F}$-fluorodeoxyglucose and ${ }^{18} \mathrm{~F}$-fluorocholine PET/CT to predict early recurrence of unifocal hepatocellular carcinoma after surgical resection. Nucl Med Commun 33:757-765

3. Selzner M, Hany TF, Wildbrett P, McCormack L, Kadry Z, Clavien PA (2004) Does the novel PET/CT imaging modality impact on the treatment of patients with metastatic colorectal cancer of the liver? Ann Surg 240:1027-1036

4. Kim YJ, Yun M, Lee WJ, Kim KS, Lee JD (2003) Usefulness of ${ }^{18}$ F-FDG PET in intrahepatic cholangiocarcinoma. Eur J Nucl Med Mol Imaging 30:1467-1472

5. Kuhn FP, Crook DW, Mader CE, Appenzeller P, von Schulthess GK, Schmid DT (2013) Discrimination and anatomical mapping of PET-positive lesions: comparison of CT attenuation-corrected PET images with coregistered MR and CT images in the abdomen. Eur J Nucl Med Mol Imaging 40:44-51

6. Delbeke D, Martin WH, Sandler MP, Chapman WC, Wright JK Jr, Pinson CW (1998) Evaluation of benign vs malignant hepatic lesions with positron emission tomography. Arch Surg 133: 510-516

7. Magini G, Farsad M, Frigerio M, Serra C, Colecchia A, Jovine E, Vivarelli M, Feletti V, Golfieri R, Patti C, Fanti S, Franchi R, Lodi F, Boschi S, Bernardi M, Trevisani F (2009) C-11 acetate does not enhance usefulness of F-18 FDG PET/CT in differentiating between focal nodular hyperplasia and hepatic adenoma. Clin Nucl Med 34:659-665

8. Cerci JJ, Pereira Neto CC, Krauzer C, Sakamoto DG, Vitola JV (2013) The impact of coaxial core biopsy guided by FDG PET/CT in oncological patients. Eur J Nucl Med Mol Imaging 40:98-103

9. Wang LM, Jani AR, Hill EJ, Sharma RA (2013) Anatomical basis and histopathological changes resulting from selective internal radiotherapy for liver metastases. J Clin Pathol 66:205-211 